\title{
Effects of Social Support on Psycho-Social Adjustment of Patients with Breast Cancer in Setting of Lahore Pakistan夥
}

\author{
Sadia Ashraf ${ }^{1}$, Iffat Ambreen ${ }^{2}$ and Muslim Shah*3 \\ ${ }^{1}$ Nursing Instructor, Saida Waheed FMH College of Nursing, Pakistan \\ ${ }^{2}$ Senior Nursing Instructor, Saida Waheed FMH College of Nursing, Pakistan \\ ${ }^{3}$ Assistant Professor, Rehman College of Nursing, Peshawar, Pakistan
}

Received: March 08, 2018; Published: March 27, 2018

*Corresponding author: Muslim Shah, Assistant Professor, Rehman College of Nursing, Peshawar, KP, Pakistan; Email: muslimshah266@gmail.com

\section{Abstract}

Background: Although the cancer is the disease of geriatrics but it can also occur in young age. Woman who are the younger than 40 years faces the additional challenges. Receiving the news of diagnosis of breast cancer is shocking for a female and this is one of most distressing event that a female experience. One of the major concerns related to physical self concept is their body image. Their body image is most important aspect that has been challenge by breast cancer. Such as they feels unattractive, missing their breast, coping with weight change and hair loss. They feel tired all the time; they face discrimination in their working and social environment. These factors or conditions lead to chronic stress, anxiety and depression and adversely affect the prognosis of cancer patients.

Objectives: This study focus on the social issues faced by patients with breast cancer, understanding social support in context of patients with breast cancer. It also investigates the effects of social support in adjustments of patients with breast cancer.

Method: A descriptive cross sectional study conducted on patients of breast cancer. Data was collected through questionnaire from the different cancer hospital of Lahore. Data were collected for study through Self administered questionnaire. Demographic Information form, multidimensional social support scale (MSSS) and psychosocial adjustment to illness scale self reflection (PAIS-SR) were used as research tool to collect data. A sample of 100 patients was selected by using convenient sampling method. Descriptive statistics, chi Square and Regression correlation were used to evaluate data.

Results: Results showed that there was a significant correlation between the social support scale and psychosocial adjustment scale. Analysis of 100 patients $(n=100)$ of breast cancer done. In this study psychosocial adjustment to illness scale self reflection (PAIS-SR) scales showing overall mean score of $114.75 \pm 9.36$ in all sub-classes and the total mean score of $41.48 \pm 11.04$ in multidimensional social support scale (MSSS).

Conclusion: On the basis of the results and the research questions that have mentioned earlier it has been explored that breast cancer patient's need social support for their better adjustment. It is concluded that although the females with breast cancer has faced many challenges in life but if proper support and care is given to them they can easily adjust in society.

Keywords: Breast Cancer; Social Support; Psychosocial Adjustment

Abbreviations: MSSS: Multidimensional Social Support Scale; PAIS-SR: Psychosocial Adjustment To Illness Scale Self Reflection

\section{Introduction}

Being diagnosed with breast cancer has effect on the life of a female. Good quality of life has different aspects which include physical, social, psychological aspects which has individual effect on quality of life. Social support that is provided in all these aspects of health has beneficial effects on breast cancer treatment. Women with small network of social support are at greater risk than the females who have strong social networking. According to Global Health Estimate, it is presented that worldwide approximately 50, 8000 female died due to breast cancer in year 2011. It is estimated in GLOBCAN 2008 that $50 \%$ of deaths in developed countries occur due to breast cancer and about 58\% occur in less developed world. Cancer rate is greater in less developed countries so there is an 
urgent need to effectively develop new strategies and approaches for early diagnosis, and treatment of breast cancer that be affordable and acceptable to patients of breast cancer.

The difference in breast cancer incidence rate between developed and developing countries is due to their life styles changes which include late childbirth, lower parity of females, shorter breast feeding time. This adoption of modern life style in low income countries is an important determinant for increasing the incidence of breast cancer in these countries. Incidence rate of breast cancer is higher in developing countries due to lack of early detection, lack of awareness/knowledge about disease process and lack of access to medical facilities. According to Omar Aftab (National coordinator, Pink Ribbon) it is estimated that prevalence of breast cancer in Pakistan is high .One of every nine females suffering from breast cancer. It is estimated that among all type of cancer breast cancer is highest that is 38.5\%.It is estimated that breast cancer has high prevalence rate in Pakistan every year 40,000 females dies due to breast cancer. Patients diagnose with breast cancer facing the challenges to live full and meaningful lives. Disturbed body image, womanhood adds even greater complexities that often others people of society can't imagine [1].

Physical changes like loss of breast, scars that come from surgical treatment, loss of hair, weight gain or loss feel a woman ashamed and afraid that other will reject them and feel pity on her. Feeling bad about body image also lower their sexual drive towards their partners. Woman diagnose with breast cancer can also face real anxiety, stress and emotional depression due to these changes in her body. This can be even harder for loved ones as well have difficulty dealing with changes that come in patient's life. Breast cancer patient's experiences lack of social support, adjustment problems in their daily life routines [2]. Woman who experience disturbed body image, may feel her body disfigured if she has lost part of their female identity. Women have sexual problems, disturbed social functioning. Minority of females have problems in performing their activities of daily living and performing task at their workplace if they are employed. Discrepancy in level to adjustment problems might be somewhat explain by related to disease and socio-demographic factors, especially: stage of disease, number of affected lymph nodes, and type of surgery, age, socioeconomic status, education and marital status.

Chronic illness and disability has profound impact on the patient's daily living pattern. Due to psychosocial adaptation illness is integrated into patient life, help to cope with disturb body image; improve self identity and self image. In psychosocial adjustment a person with disablement moves towards state of enablement, also transformation of negative feelings and well-being to positive feelings and well-being. Illness causes psychosocial dysfunction this mean be deficient in of development of psychosocial self along with other dysfunctions that may be physical, emotional and cognitive in nature. Breast cancer is a major life event. Experience of patient with breast cancer depends on her relationship with her intimate partner and on her family member. Due to disease condition not only the patient but also the family and intimate partner experience depressed mood and other psychosocial adjustment problems. Individual and other relationship factors, e.g. couple's joint efforts to deal with cancer are likely to contribute their psychosocial adjustment in society. For their adjustment they need more knowledge about their disease process and knowledge about their adjustment problems and how to deal with problems.

According to national academic press survey on patients with cancer experience anxiety on the day of their diagnosis, Depression, Cancer related post-traumatic stress and psychosocial distress existing ranging from normal adjustments issues to major adjustments disorders. For patient diagnosed with breast cancer anxiety can also intensify the anticipation of pain, and other symptoms of pain like sleep disturbance .Anxiety can significantly interfere with quality of life of patients with breast cancer and their family members and this should be evaluate and treated for their adjustments NAP, 2004. When a patient learns that she has breast cancer, she is in phase of shock. She may feel that her life has been turned upside down. But after sometime when phase of shock wears off, the process of making changes in her life begins. Patient thinks about the treatment plan and she has to re-arrange things in her daily living and at her workplace. She may have to find innovative ways to talking about the disease to her loved one and health care providers.

\section{Methodology}

A quantitative descriptive cross sectional study was conducted on 100 patients of breast cancer aged between 15-65 years. In this study the research subjects were the patients of breast cancer taken from different hospitals of Lahore. Lahore has the population of 7.5 million. If we want to compute the number of female population in Lahore then we have to: $7500,000 / 2=37500,000$. According to Formula for calculating the sample size is [3].

$$
n=\frac{N}{1+N(e)^{2}}
$$

While $\mathrm{n}$ is the sample size, $\mathrm{N}$ represents the population and $\mathrm{e}$ is the level of precision in this formula. From the Yamani table if we take the level of precision $10 \%$ then we put the values in the table

$$
\begin{gathered}
=\frac{37500.000}{1+37500.000(0.1) 2} \\
n=99.99
\end{gathered}
$$

The research study was authorized by Ethical Committee and Advance Study Research Board of the Institute Of Social and Cultural studies Public Health Department University of the Punjab Lahore. A notified approval and permission was taken from the research center at Shokat Khanum Memorial Cancer Hospital Lahore and Cancer ward of Mayo Hospital Lahore. Participants were provided with information about research in order to obtain consent. It was achieved through a letter of consent attached to each questionnaire stating the basis of the research, duration of participation, confidentiality as well as benefits. In addition permission of the research was taken by ethical review committee of the university on a letter. In this regard, before carrying out study, necessary permissions were obtained from Ethical Committee of hospital where study will be conducted. 


\section{Data Collection Instruments}

Data was collected through descriptive information form, Multidimensional social support scale (MSSS) and Psychosocial adjustment to illness scale (PAIS) were used to collect data.

\section{The Descriptive Information Form}

Consist of descriptive questions from participants such as age, educational status, marital status, occupation, income level, place of residence, stage of cancer, and type of surgical treatment.

\section{Multidimensional Social Support Scale}

This scale was used by Zimet, Dahlem, and Zimet \& Farley in 1988 in their study [4]. Researcher has adopted it is to identify the social support level in breast cancer patients. The Multidimensional Scale of Social Support (MSSS) is a brief research tool designed to measure perceptions of support from 3 sources: Family, Friends, and a Significant Other. The scale is comprised of a total of 12 questions to identify level of social support.

\section{Psychosocial Adjustment To Illness Scale Self Reflection (PAIS-SR)}

Psychosocial adjustment to illness scale used and developed by Derogates, Leonard R Lopez in 1983 in their study on cancer patient in setting of Turk. I have used this scale as it is to collect data from breast cancer patient in Lahore, Pakistan setting. The psychosocial adjustment to illness scale Self reflection (PAIS-SR) included 46 items and their subtypes are further included different items e.g. Health care orientation (8 items), Vocational environment (6 items), Domestic environment (8 items), Sexual Relationship (6 items), Family Relationship (5 items), Social environment (6 items), Psychological distress (7 items).This scale is designed to assess the patient's psychosocial adjustment to medical illness.

\section{Results}

According the results received the mean age of research participants was 26 - 35 years. It was observed that there were 32 breast cancer patients (32\%) belong to this class of age group. Out of the total 100 patients, there were 68 percent $(n=68)$ of the population married from the whole. It was observed that there $27 \%$ of participants were high school graduates, $39 \%$ were house wives, $57 \%$ of participants were living in joint family system. Approximately half of population (53\%) was living in cities, 57\% of participant's reports that their income is too much less than expenditure. Nearly $36 \%$ of subjects were in their III stage of their disease process. Whereas $42 \%$ of females have undergone the mastectomy, $12 \%$ of them have gone through breast conserving surgery. While $40 \%$ have not received any type of surgery.

\section{Demographic Data of Patients N=100}

A multidimensional social support scale was used as like rt scale to signify the feelings of the patients about the any kind of multidimensional social support according to the statements in the each of the question. There were 12 items in this scale and all the study participants were have to give answer of these statements. In this table the cumulative mean overall value of this scale was 41.48 \pm 11.05 according to the parameters set already for measurements.

\section{Psychosocial Adjustment to Illness Scale}

The coefficient alpha for the total PAIS-SR scale in our study was noticed 0.93 and the range of the sub-scales were from 0.77 to 0.95 .

\section{Psychosocial Adjustment of the Breast Cancer Patients with Respect to the Average of Md-SSS ( Multidimensional Social Support Scale) Score}

The average scores of multidimensional social score support was measure in respect to have the values of chi-square and p-value with psychosocial adjustment to illness scale-self report. The calculated chi-square value was 36.91 and the p-value was 0.001 .

Multidimensional Social Support Scale Scores and Psychosocial Adjustment to Illness Scale Scores for the Breast Cancer Patients [Correlation (Spearman)]

The spearman correlation was calculated between multidimensional social support scale score with psychosocial adjustment to illness score self-report given down in the table. The mean score was calculated and the values were sorted out in both positive and negative significance. The calculation were determined by spearman correlation and with the help of Spearman's Rankorder correlation (rho) the coefficient of each of the class of psychosocial adjustment to illness scale ( $r$ ) was calculated. The value of coefficient of correlation ( $r$ ) shows the strength of the association of the various factors under study by determining the p-value at different stages. Such as in some of the variables like; sexual support and social environment were in a negative value because the value of coefficient of correlation ( $r$ ) varies from -1 to +1 . According to the result the calculation was done and means were compared by two tailed test hence the p-value less than 0.05 shows the positive association between the various factors under study. All of the variables in the psychosocial adjustment to illness scale were associated to multidimensional social support scale of cancer among all of the cancer patients under study. The strength of the association is shown by coefficient of correlation ( $r$ ) and the association is shown by p-value of these variables.

\section{Level of Education and Psychological Adjustment to Illness Scale - Self Report}

A comparison was done between the level of education and PAIS-SR to define the relationship of education with this scale. A two by two contingency table was drawn and by cross tabulation the value of chi-square and p-value was find out. The following table shows the best illustration of this relationship. The results showed there was a positive association between the educational status of the study participants and the psychosocial adjustment scale-self report solved by the patients under study.

\section{Significance of Occupation of Study Participants with Psychological Adjustment to Illness Scale - Self Report}

The significance of the occupation of all of the study participants was compared with the psychosocial adjustment to illness scale to 
analyze the association between these two factors. Below table shows the whole calculation of the results; According to the results there was not a significant relationship of the occupational status of the patients and the psychosocial adjustment to illness scale-self report by the all study participants.

\section{Influence of Type of Surgical treatment of Study Participants with Psychological Adjustment to Illness Scale - Self Report}

Cross-tabulation was done to notify the influence of the type of surgical treatment of the cancer patients with the psychosocial adjustment to illness scale-self report and the level of significance. The following table shows the calculation of this relationship;

\section{Discussion}

Diagnosis of breast cancer put a great mental strain on female's life that the other people of society can't imagine. Disturbed body image and womanhood can cause problems in psychosocial adjustments for patients. In the process of adjustment there is a significant relationship between the mind and body and with the disturbed body it is difficult to cope in the society. Involving coping and compromise, adjustment is defined as the power to accept internal and external changes and to exhibit appropriate attitudes and behaviors. The process of adjustment requires interplay between the mind and body. It is studied in different interdependent categories such as biological, developmental, psychological, socio cultural, spiritual and technological. There was a significant difference between the descriptive characteristics of the participants and their total scores in the psychosocial adjustment to illness scale in terms of educational status, occupation, place of residence, number of children, income level and type of operation.

In the present study, the time of diagnosis was not influential in adjustment. Ayaz observed that those who were high school graduates and employed with a decent economic status had higher social support scores [5]. In the present study psychosocial adjustment to illness scale self reflection (PAIS-SR) scales showing overall mean score of $114.75 \pm 9.36$ in all sub-classes. Similarly in a study conducted by Cinar [6], on the patients with hem dialysis had a mean score of $68.94 \pm 14.7$. In another study that is conducted on the patients with breast cancer by Swain had a mean score of $51.46 \pm 9.97$. The mean scores of the present study and the studies Table 1: Demographic characteristics. carried out by Cinar and by Swain (1996) are showing the huge differences, which might be giving the idea that the patients from different culture and values behave differently towards illness and the human beings who are from same culture and having same norms and values react to illness similarly across the same culture.

However the total score in this study reveals that the patient has good psychosocial adjustment. Similarly a study conducted by Butler concluded that the patients with breast cancer show good psychosocial adjustment. Another study by Cam concluded that breast cancer patients have normal psychosocial adjustment towards their disease. The similarities in the present study and the studies by Butler and Cam may be due to similarities in the Individual, regional, socio-economic factors that are affecting the adjustment process. In the present study, results show the positive psychosocial adjustment to for breast cancer patients. The correlation in PAIS scale shows that there was positive significant correlation in health care orientation, vocational environment and domestic environment domain as compare to Sexual relationship and social environment was in negative significant correlation. It is found that social support is provided by the family members mainly affects the adaptation process and has positive outcomes.

In Pakistan because there is the traditional culture and mostly joint family system the participants of this study were receiving social support mostly from their family members. Due to social support patients are more hopeful towards their good prognosis. Cinar reported that there is positive correlation between social support and health care orientation domain [6-10]. According to McDonough when social support given to patient it brings positive changes in well-being of patients as compared to stress has negative effect on well-being [10-15]. Social support and well-being was demonstrated as a significant prognostic factor for breast cancer outcomes [8-19]. It particularly facilitates one's recovery, enhances his/her adjustment to medical treatment and overall life quality, and prolongs his/her life span. Identification and reinforcement of social support systems, along with counseling when necessary, will help women with breast cancer cope with their problems and maintain their welfare. Therefore, all health staff, especially nurses who are in more frequent interactions with patients, need to identify social support levels of patients with breast cancer, and to plan and use support initiatives accordingly (Tables 1-8).

\begin{tabular}{|c|c|c|c|}
\hline \multicolumn{2}{|c|}{ Demographic characteristics } & No & $\%$ \\
\hline \multirow{5}{*}{ Age group } & $16-25$ yrs. & 1 & $1 \%$ \\
\hline & $26-35$ yrs & 32 & $32 \%$ \\
\hline & $36-45$ yrs & 30 & $30 \%$ \\
\hline & $46-55$ yrs & 17 & $17 \%$ \\
\hline & $56-65$ yrs & 20 & $20 \%$ \\
\hline \multirow{4}{*}{ Marital Status } & Single & 10 & $10 \%$ \\
\hline & Married & 68 & $68 \%$ \\
\hline & Widow & 14 & $14 \%$ \\
\hline & Divorced & 8 & $8 \%$ \\
\hline
\end{tabular}




\begin{tabular}{|c|c|c|c|}
\hline \multirow{5}{*}{ Level of education } & Literate & 21 & $21 \%$ \\
\hline & Primary school & 23 & $23 \%$ \\
\hline & High school & 27 & $27 \%$ \\
\hline & Intermediate & 25 & $25 \%$ \\
\hline & University level & 4 & $4 \%$ \\
\hline \multirow{3}{*}{ Family type } & Extended & 20 & $20 \%$ \\
\hline & Joint family & 67 & $67 \%$ \\
\hline & Nuclear & 23 & $23 \%$ \\
\hline \multirow{6}{*}{ Type of occupation } & House wife & 39 & $39 \%$ \\
\hline & Civil servant & 4 & $4 \%$ \\
\hline & Self employed & 13 & $13 \%$ \\
\hline & Retired & 1 & $1 \%$ \\
\hline & Private & 29 & $29 \%$ \\
\hline & Others & 14 & $14 \%$ \\
\hline \multirow{3}{*}{ Level of income } & $<$ than expenditure & 57 & $57 \%$ \\
\hline & Equal to & 24 & $24 \%$ \\
\hline & expenditure $>$ than expenditure & 19 & $19 \%$ \\
\hline \multirow{3}{*}{ Place of residence } & Village & 26 & $26 \%$ \\
\hline & Town & 21 & $21 \%$ \\
\hline & City & 53 & $53 \%$ \\
\hline \multirow{4}{*}{ Stage of disease } & Stage I & 16 & $16 \%$ \\
\hline & Stage II & 30 & $30 \%$ \\
\hline & Stage III & 36 & $36 \%$ \\
\hline & Stage IV & 18 & $18 \%$ \\
\hline \multirow{4}{*}{ Type of treatment } & Breast conserving & 12 & $12 \%$ \\
\hline & surgery & 42 & $42 \%$ \\
\hline & Mastectomy & 40 & $40 \%$ \\
\hline & Received no surgery Others & 6 & $6 \%$ \\
\hline
\end{tabular}

Table 2: Psychosocial Adjustment to Illness Scale.

\begin{tabular}{|c|c|c|}
\hline Variables & Items & Mean \pm SD \\
\hline Health Care Orientation & 08 & $19.64 \pm 3.21$ \\
\hline Vocational Environment & 06 & $16 \pm 2.31$ \\
\hline Domestic Environment & 08 & $17.92 \pm 2.63$ \\
\hline Sexual Relationship & 06 & $14.29 \pm 1.65$ \\
\hline ExtendedFamily Relationship & 05 & $11.90 \pm 1.67$ \\
\hline Social Environment & 06 & $14.50 \pm 1.63$ \\
\hline Psychological Environment & 07 & $20.64 \pm 3.10$ \\
\hline Total PAIS-SR & 46 & $114.75 \pm 9.36$ \\
\hline Multidimensional Social Support Scale Score & 12 & $41.39 \pm 6.58$ \\
\hline
\end{tabular}

Table 3: Psychosocial Adjustment of the Breast Cancer Patients with Respect to the Average of MD-SSS ( Multidimensional Social Support scale) Score.

\begin{tabular}{|c|c|c|c|c|c|c|}
\hline \multirow{2}{*}{ MDSSS } & \multicolumn{2}{|c|}{ Psychosocial adjustment to Illness Scale -Self Report } & \multirow{2}{*}{ Total } \\
\cline { 2 - 6 } & Poor & \% age & Good & \% age & \\
\hline Below average & 44 & 44 & 06 & 06 & 44 \\
\hline Above average & 24 & 24 & 26 & 26 & 56 \\
\hline Total & 68 & 68 & 32 & 32 & 100 \\
\hline
\end{tabular}


Table 4: Psychosocial Adjustment to Illness Scale.

\begin{tabular}{|c|c|c|c|c|c|c|}
\hline Items No & S. Agree & Disagree & Neutral & Agree & S.Agreee & Mean \pm SD \\
\hline 1. & 06 & 38 & 20 & 30 & 06 & $2.92 \pm 1.07$ \\
\hline 2. & 11 & 33 & 25 & 24 & 07 & $2.83 \pm 1.12$ \\
\hline 3. & 02 & 06 & 07 & 49 & 36 & $4.11 \pm 0.92$ \\
\hline 4. & 06 & 17 & 30 & 39 & 08 & $3.26 \pm 1.03$ \\
\hline 5. & 07 & 37 & 30 & 20 & 06 & $2.81 \pm 1.03$ \\
\hline 6. & 07 & -- & 27 & 61 & 05 & $3.64 \pm 0.68$ \\
\hline 7. & 01 & 12 & 43 & 36 & 08 & $3.38 \pm 0.83$ \\
\hline 8. & 1 & 4 & 5 & 43 & 47 & $4.31 \pm 0.82$ \\
\hline 9. & -- & 11 & 33 & 51 & 5 & $3.50 \pm 0.75$ \\
\hline 10. & 9 & 35 & 28 & 18 & 10 & $2.85 \pm 1.13$ \\
\hline 11. & 1 & 4 & 12 & 47 & 36 & $4.13 \pm 0.85$ \\
\hline 12. & 1 & 7 & 30 & 50 & 12 & $3.65 \pm 0.82$ \\
\hline
\end{tabular}

Table 5: Multidimensional Social Support Scale Scores and Psychosocial Adjustment to Illness Scale Scores for the Breast Cancer Patients [Correlation (Spearman)].

\begin{tabular}{|c|c|c|}
\hline \multirow{2}{*}{ PAIS-SR Score } & \multicolumn{2}{|c|}{ MD-SSS } \\
\cline { 2 - 3 } & $\mathbf{r}$ & $\mathbf{p}$-Value \\
\hline Health Care Orientation & 0.900 & 0.001 \\
\hline Vocational Environment & 0.267 & 0.007 \\
\hline Domestic Environment & 0.300 & 0.002 \\
\hline Sexual Relationship & -0.221 & 0.07 \\
\hline Extended Family Relationship & 0.379 & 0.001 \\
\hline Social Environment & -0.222 & 0.026 \\
\hline Psychological Environment & 0.329 & 0.001 \\
\hline
\end{tabular}

Table 6: Level of Education and Psychological Adjustment to Illness Scale - Self Report.

\begin{tabular}{|c|c|c|c|c|c|}
\hline \multirow{2}{*}{$\begin{array}{l}\text { Education Level of the Study } \\
\text { Participants }\end{array}$} & \multicolumn{2}{|c|}{ Psychosocial Adjustment to illness } & \multirow{2}{*}{ Total } & \multirow{2}{*}{ Chi-Square } & \multirow{2}{*}{ p-Value } \\
\hline & Poor & Good & & & \\
\hline Literate & 14 & 7 & 21 & \multirow{5}{*}{4.180} & \multirow{5}{*}{0.03} \\
\hline Primary & 12 & 11 & 23 & & \\
\hline High School & 21 & 6 & 27 & & \\
\hline Intermediate & 18 & 7 & 25 & & \\
\hline University & 3 & 1 & 4 & & \\
\hline Total & 68 & 32 & 100 & & \\
\hline
\end{tabular}

Table 7: Significance of Occupation of Study Participants with Psychological Adjustment to Illness Scale - Self Report.

\begin{tabular}{|c|c|c|c|c|}
\hline \multirow{2}{*}{ Occupation of Study Participants } & \multicolumn{2}{|c|}{ Psychosocial Adjustment to Illness } & \multirow{2}{*}{ Total } & \multirow{2}{*}{ Chi-Square } \\
\cline { 2 - 4 } & Poor & Good & 39 & \\
\hline House Wife & 26 & 13 & 4 \\
\hline Civil Servant & 3 & 1 & 13 & \multirow{2}{*}{6.233} \\
\hline Self Employed & 7 & 0 & 29 & \\
\hline Retired & 1 & 11 & 14 \\
\hline Private Employ & 18 & 1 & 100 \\
\end{tabular}


Table 8: Influence of Type of Surgical treatment of Study Participants with Psychological Adjustment to Illness Scale - Self Report.

\begin{tabular}{|c|c|c|c|c|}
\hline \multirow{2}{*}{ Type of Surgical Treatment } & \multicolumn{2}{|c|}{ Psychosocial Adjustment To Illness } & \multirow{2}{*}{ Total } & \multirow{2}{*}{ Chi-Square } \\
\cline { 2 - 4 } & Poor & Good & 12 & \\
\hline Breast Conserving Surgery & 11 & 1 & 42 & \multirow{2}{*}{25.84} \\
\hline Mastectomy & 17 & 25 & 40 & \multirow{2}{*}{0.001} \\
\hline Received no Surgery & 34 & 6 & 6 & \\
\hline Others & 6 & 0 & 100 & \\
\hline Total & 68 & 32 & \\
\hline
\end{tabular}

\section{Conclusion}

In this study it was concluded that patients of breast cancer need social support for their better adjustment. Social support had important effect on the adjustment with their disease. For the better adjustment provide holistic care to patients, and this care not only provided by medical and paramedical staff but also from the family members. It is concluded that family should be involved in the treatment process; they have complete knowledge about the disease status of patient. They should have knowledge that patient of breast cancer not only suffering from physical disability but also from mental strain, so that why they also need psychological support from their loved ones. Patients of breast cancer should encourage taking part in different social activities that they were doing before their disease. It is concluded that level of education, place of residence, type of treatment, income level, and type of family has important role in psychosocial adjustment of patient. Patient who are educated and high economic status have high level of social support. It is found that patient with joint family system and who have orientation to health had good social support level as compared to those who have not. They are more actively involved in social activities in doing their household work so that they have less psychological problems. It is concluded that family must have up to date knowledge of disease and should also be involved in treatment process. Patients who get emotional support from their partners have higher score in psychosocial adjustment.

\section{Limitations of study}

Limitations are the matters that happen or occur in the study and those are out of researchers controlled. Every study has some limitation in it.

\section{Limitation of this study is that the}

a. Data was collected from the patients that were in the hospital setting not from the social environment or community setting.

b. Only two hospitals were selected for data collection.

c. Sample size should be large and generalizable.

d. In this study those patients of breast cancer was selected who just had diagnosis of breast cancer no matter what was the duration from of diagnosis.

e. Patients should be selected who had at least 6 month of diagnosis.

\section{References}

1. King KE (2000) Writing the Breast Cancer Joumev: Illness Narratives fiom an Intemet Forum: pp. 124-130.

2. Helms RL, O Hea EL, Corso M (2008) Body image issues in women with breast cancer. Psychology Health and medicine 13(3): 313-325.

3. Yamane Taro (1967) Statistics: An Introductory Analysis, 2nd Ed, New York: Harper and Row: pp. 919.

4. Zimet, GD, Dahlem NW, Zimet SG, Farley GK (1988) The multidimensional scale of perceived social support. Journal of personality assessment 52(1): 30-41.

5. Weihs KL, Enright TM, Simmens SJ (2008) Close relationships and emotional processing predict decreased mortality in women with breast cancer: preliminary evidence. Psychosomatic medicine 70(1): 117-124.

6. Cinar S (2009) Psychosocial adjustment and social support in hemodialysis patients. Türkiye Klinikleri J Nurs Sci 1: 12-18.

7. McDonough MH, Sabiston CM, Wrosch C (2014) Predicting changes in posttraumatic growth and subjective well-being among breast cancer survivors: the role of social support and stress. PsychoOncology, 23: 114-120.

8. Susan G Komen (2013) Benefits of Social Support, USA.

9. Alferi SM, Carver CS, Antoni MH, Weiss S, Duran RE (2001) An exploratory study of social support, distress, and life disruption among low-income Hispanic women under treatment for early stage breast cancer. Health Psychology 20(1): 41.

10. Aliaga M, Gunderson B Interactive Statistics.

11. American cancer society (2015) Breast cancer types.

12. Bloom JR, Stewart SL, Johnston M, Banks P, Fobair P (2001) Sources of support and the physical and mental well-being of young women with breast cancer. Social science \& medicine 53(11): 1513-1524.

13. Derogatis LR (1986) The psychosocial adjustment to illness scale (PAIS). Journal of psychosomatic research 30(1): 77-91.

14. Lahore Nama (2015) The capitals of Pakistan: Lahore.

15. Merriam Webstar (2015) Psychosocial, USA.

16. MacMahon B, Cole P, Brown J (1973) Etiology of human breast cancer: a review. Journal of the National Cancer Institute 50(1): 21-42.

17. Preston M (2010) An Exploration of Appearance-Related Issues of Breast Cancer Treatment on Sense of Self, Self-Esteem, and Social Functioning in Women with Breast Cancer. Spring pp. 5-170.

18. Segrin, Badger T, Dorros SM, Meek P, Lopez AM (2007) Interdependent anxiety and psychological distress in women with breast cancer and their partners. Psycho-Oncology 16(7): 634-643.

19. Taylor SE (2011) Social support: A Review. In: MS Friedman. The Handbook of Health Psychology:: Oxford University Press, New York, USA, pp. 189. 
(c) (i) This work is licensed under Creative

Submission Link: https://biomedres.us/submit-manuscript.php

Assets of Publishing with us
$\begin{aligned} & \text { BIOMEDICAL } \\ & \text { RESEARCHES }\end{aligned}$

